\title{
Disorientation Angle Distribution of Primary Particles in Potash Alum Aggregates
}

\author{
Tijana Kovačevića ${ }^{\text {, Viktoria Wiedmeyer }}{ }^{\mathrm{b}}$, Jonathan Schock $^{\mathrm{c}}$, Andreas Voigt ${ }^{\mathrm{b}}$, \\ Franz Pfeiffer ${ }^{\mathrm{c}}$, Kai Sundmacher ${ }^{\mathrm{b}, \mathrm{d}}$, Heiko Briesen ${ }^{\mathrm{a}}$ \\ ${ }^{a}$ Chair of Process Systems Engineering, Center for Life and Food Sciences Weihenstephan, \\ Technical University of Munich, 85356 Freising, Germany \\ ${ }^{b}$ Chair of Process Systems Engineering, Otto-von-Guericke Universität Magdeburg, 39106 \\ Magdeburg, Germany \\ ${ }^{c}$ Chair of Biomedical Physics, Department of Physics, Technical University of Munich, \\ 85748 Garching, Germany \\ ${ }^{d}$ Max Planck Institute for Dynamics of Complex Technical Systems, 39106 Magdeburg, \\ Germany
}

\begin{abstract}
In order to fully characterize crystal aggregates, the orientation of primary particles has to be analyzed. A procedure for extracting this information from three-dimensional microcomputed tomography $(\mu \mathrm{CT})$ images was recently published by our group. We here extend this method for asymmetrical crystals and apply it for studying the disorientation angle distribution of four potash alum crystal samples that were obtained under various experimental conditions. The results show that for all considered supersaturation profiles, primary particle pairs tend to have the same orientation significantly more often than in theoretical considerations, in which the orientations of primary particles are assumed to be distributed randomly.
\end{abstract}

Keywords: A1. Aggregation, A1. Characterization, A1. Microcomputed Tomography, A1. Crystal Morphology, B1. Salts

\section{Introduction}

Aggregation is an important phenomenon in crystallization and can lead to broadening of the particle size distribution, an undesired change in flow properties, and the inclusion of impurities 1 [p. 25]. In an agitated vessel, crystals often collide and form an aggregate if they are cemented together quickly enough 
to survive the hydrodynamic forces attempting to separate them [2, 3, 4]. The rate at which the particles are bound together depends on, among other factors, the type of contact between the particles [4, which further depends on the shape of particles and their mutual orientation. These aspects have typically been simplified by considering a point or linear contact between spherical particles, as reviewed by Hounslow et al. 4]. Shape information has so far been included in the aggregation modeling through a shape-sensitive aggregation kernel [5] or by adopting a Monte Carlo approach and a hierarchical aggregate representation [6, 7.

The need for a more detailed understanding of the ability of a particle pair to form a stable aggregate requires a technique to characterize the particles in terms of their mutual orientation. This topic is of special importance in nanoand bio-materials, where oriented particle attachment occurs $[8]$. Collier et al. [9, 10] investigated calcite aggregates using a transmission electron microscope (TEM) and a scanning electron microscope (SEM). A TEM enables measuring diffraction patterns, which can be used to analyze the aggregate in terms of the alignment between the primary particles. A SEM enables simple observation, and no alignment measurements can be performed. In [10, the alignment measured within an aggregate was classified as "perfect", "almost perfect", "partial" and "no alignment". $40 \%$ of the particles were classified into the first two classes for an experiment conducted at low ionic strength. Crystals produced at a higher ionic strength were imaged using an SEM and no alignment was visually observed. Collier et al. [10] explained the observed behavior by assuming that crystals grown at a lower ionic strength have enough time to reorient themselves into an energetically more favorable position before being cemented together. This time window is further explained by the increase in thickness of the electrical double layer that, "[...] either slows the approach of the crystals, allowing them to rotate before cementing, or holds them far enough apart that only those colliding in favorable orientations are bound strongly enough to withstand the hydrodynamic forces in the vessel" [10].

Apart from the TEM studies, information about the crystal shape can be 
obtained using image processing. Classification techniques such as discriminant factorial analysis or support vector machine can be combined with twodimensional (2D) image-based shape factor analysis to measure the degree of agglomeration [11, 12, 13, or to track the aggregate volume [14. Threedimensional (3D) and stereo imaging techniques enable a full description of the particle shape [15, 16, 17, 18, 19]. They can be used to obtain the size, shape, and orientation of each primary particle in an aggregate [20. In the present work, we apply our approach to extracting such information, presented in [20], to potash alum crystals grown under various experimental conditions. We wish to determine whether supersaturation influences the orientation between primary particles in an aggregate. Potash alum was chosen for reasons of simplicity, being a model compound that crystallizes into octahedra. It has been shown experimentally that potash alum forms stronger agglomerates at higher supersaturation levels [21. This compound is known to exhibit parallel growth and twinning [1][p. 25], which can lead to an increased amount of identically oriented primary particles in an aggregate.

Describing a set of crystallites in terms of their mutual orientation is a wellknown problem of material science [22]. The orientation between the crystallites in the polycrystalline material can influence its properties, such as in which direction the material is especially magnetizable [22][p. 1]. Thus, we adopt here the concepts of disorientation angle and disorientation angle distribution (DAD) from material science. We compare our experimentally measured values to the case considered in the literature [23, 24, where each primary particle orientation is equally probable.

\section{Description of Crystal Shape}

As in our previous publications [16, 20, 25], we consider three crystal representations. A $V$-representation consists of a matrix $\mathbf{V}$ of crystal vertices $\mathbf{v}_{i}$. A crystal can be analogously described by a set of face normals $\mathbf{a}_{i}$ from the crystal middle point, gathered as rows in the matrix A. This matrix, along 
with the vector $\mathbf{h}$ of crystal face distances $h_{i}$ from some middle point, forms the $H$-representation. Given the crystal symmetry, the dimension of the vector $\mathbf{h}$ can be reduced by considering only one face distance $h_{i}$ per crystal face group to obtain the face distance vector $\mathbf{h}_{\mathrm{C}}$. This is referred to as $H_{C}$-representation.

In the following, bold symbols represent vectors and matrices, and italic symbols represent scalar values. Indices that are neither bold nor italic indicate a name or a description. Furthermore, we use "aggregate" as a general term for a crystal consisting of more than one primary particle. The word "agglomerate" refers to strongly bound aggregates in which primary particles are grown into each other. Depending on the context, "crystal" may refer to either a single crystal or an aggregate.

\section{Experiments}

\subsection{Crystallization Experiments}

We conducted two sets of potash alum seeded batch cooling crystallization experiments. The experimental parameters are given in Table 1. The bulk material was purchased from Merck (CAS No. 7784-24-9, assay 99.0-100.5 \% calc. on dry substance). In order to ensure saturation at $30{ }^{\circ} \mathrm{C}, 161 \mathrm{~g}$ of potash alum was dissolved in $1000 \mathrm{~g}$ of deionized water (161.2 g - $161.3 \mathrm{~g})$. The $1 \mathrm{l}$ double-jacketed vessel with an inner diameter of $10 \mathrm{~cm}$ and a round bottom was stirred at $250 \mathrm{rpm}$. The dispersion was heated above the saturation temperature to ensure complete dissolution and then cooled at the cooling rates given in Table 1. The seed material was purchased from either Roth (Art. Nr. CN78.2) or Merck. It was sieved for 60 min with a vibratory sieve shaker (AS 200 control, Retsch). The sieve fraction between $200 \mu \mathrm{m}$ and $300 \mu \mathrm{m}$ was added when the temperature was slightly below the calculated saturation temperature. Crystals were imaged using an online flow-through-cell QICPIC (R02, Sympatec) system, as previously described in [26, 27.

As it can be seen in Table 1, the M experiments were performed with different seeds and a larger amount of seed than in experiment $\mathrm{R}$ in order to 


\begin{tabular}{|c||c||c|c|c|}
\hline Param./Name & $\mathrm{R}$ & $\mathrm{M} 10$ & $\mathrm{M} 5$ & $\mathrm{M} 3$ \\
\hline \hline Cooling rate & $5 \mathrm{~K} / \mathrm{h}$ & $10 \mathrm{~K} / \mathrm{h}$ & $5 \mathrm{~K} / \mathrm{h}$ & $3 \mathrm{~K} / \mathrm{h}$ \\
\hline Seed material & Roth & Merck & Merck & Merck \\
\hline Seed mass & $8 \mathrm{~g}$ & $10 \mathrm{~g}$ & $10 \mathrm{~g}$ & $10 \mathrm{~g}$ \\
\hline Seeding temp. & $29.87^{\circ} \mathrm{C}$ & $29.84^{\circ} \mathrm{C}$ & $29.76{ }^{\circ} \mathrm{C}$ & $29.82{ }^{\circ} \mathrm{C}$ \\
\hline End temp. & $20.4^{\circ} \mathrm{C}$ & $21.3{ }^{\circ} \mathrm{C}$ & $20.8{ }^{\circ} \mathrm{C}$ & $20.5{ }^{\circ} \mathrm{C}$ \\
\hline Effective voxel size & $6.80 \mu \mathrm{m}$ & $7.85 \mu \mathrm{m}$ & $7.85 \mu \mathrm{m}$ & $7.85 \mu \mathrm{m}$ \\
\hline Preparation & Aggregates & All & All & All \\
\hline
\end{tabular}

Table 1: Parameters of the batch cooling crystallization experiments.

ensure more agglomeration and less nucleation. The results of the preliminary processing on the experiment $\mathrm{R}$ were presented at the BIWIC 2016 conference 28] 11 Furthermore, two different preparation procedures were performed and different $\mu \mathrm{CT}$ resolutions were used, as explained below. In $\mathrm{M}$ experiments, different constant cooling rates were applied to obtain different supersaturation profiles while the other parameters were kept constant. This was done because supersaturation is expected to have an influence on the DAD.

At the end of the experiments, crystals were withdrawn from different locations in the crystallizer using a large pipette and placed on a Büchner funnel covered by filter paper. Each sample obtained this way was filtered using a vacuum pump, washed with a cooled 50-50 solution of ethanol-water, and air dried.

The temperature was measured with a PT100 sensor and the concentration was monitored using an ATR-FTIR probe (Nicolet iS 10 FT-IR, Thermo Fisher Scientific). Spectra for a potash alum solution in the considered temperature and concentration range were used for a partial least squares calibration model.

\footnotetext{
${ }^{1}$ Processing was repeated with the user-assisted manipulation of concavity points and the size and position of the bars in the resulting graph was slightly changed to be the same for all experiments.
} 
During the experiments, spectra were automatically recorded and evaluated with the calibration model. There is an offset in the resulting concentration. Therefore, we use the initially known concentration $c_{1}$ at time $t_{1}$. The final concentration $c_{2}\left(t=t_{2}\right)$ was determined by taking three samples of the solution, filtering the samples through filter paper, and air drying for at least two days. The concentration $c$ after offset correction is obtained from the measurement without correction, $c_{\text {meas }}$, using:

$$
c(t)=c_{\text {meas }}+c_{1}+\frac{c_{2}-c_{1}}{t_{2}-t_{1}}\left(t-t_{1}\right) .
$$

The supersaturation was computed as

$$
S(T(t))=\frac{c(t)}{c_{\mathrm{sat}}(T(t))}
$$

where $c(t)$ is the concentration in grams of hydrate per kilograms of free water at time $t$, and $c_{\text {sat }}(T(t))$, the saturation concentration for temperature $T$ measured at time $t$. The saturation concentration was previously measured and interpolated to obtain the following equation:

$$
c_{\mathrm{sat}}=\left(0.18 T_{\mathrm{K}}^{2}-102.726 T_{\mathrm{K}}+14760.7\right) \frac{\mathrm{g} \text { hydrate }}{\mathrm{kg} \text { free water }},
$$

where $T_{\mathrm{K}}$ stands for the temperature in Kelvin. The saturation is in good agreement with measurements made by Mullin et al. [29]. The concentration and supersaturation curves are shown in Figures $1 \mathrm{a}$ and $1 \mathrm{~b}$,

It can be seen that the concentration increases at the beginning and then decreases for the rest of the experiment. This is consistent with the QICPIC observation that the seed crystals start dissolving upon being introduced into the reactor, as seen in Figure 2. Furthermore, the measured supersaturation was the largest in the experiment with the fastest cooling rate (M10), and the smallest in the experiment with the slowest cooling rate (M3), as expected.

\subsection{Crystal Preparation and Imaging}

In order to obtain a sample consisting only of aggregates, crystals of experiment $\mathrm{R}$ were picked up from the filter paper using a pair of tweezers, observed 


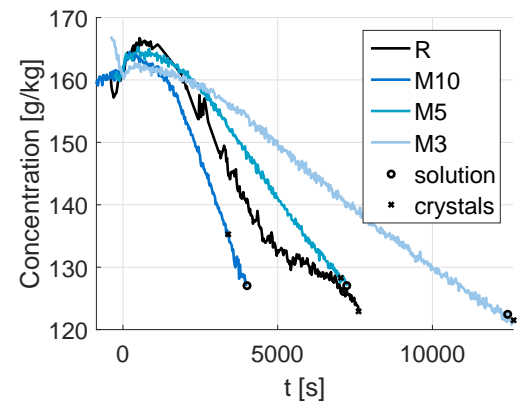

(a)

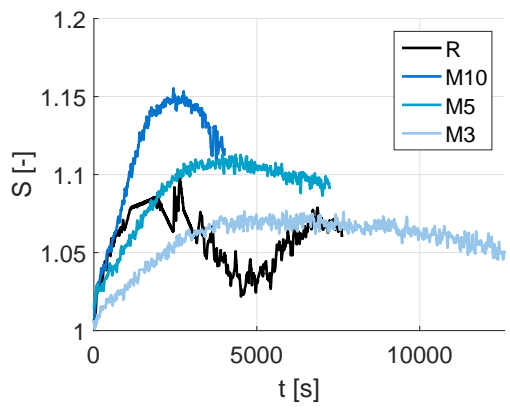

(b)

Figure 1: Concentration (a) and supersaturation (b) for all four experiments. Black circles and crosses mark the sampling time for solution and crystals, respectively. Seeds were added at time $t=0$ for all plots.

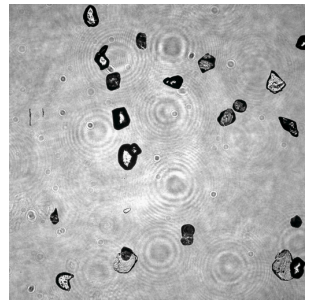

(a) $\mathrm{R}$

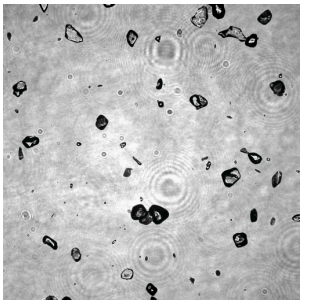

(b) M3

Figure 2: Frames from the QICPIC videos taken $37 \mathrm{~s}$ after adding seeds in the experiments $\mathrm{R}$ (a) and $270 \mathrm{~s}$ after adding seeds in experiment M3 (b). 


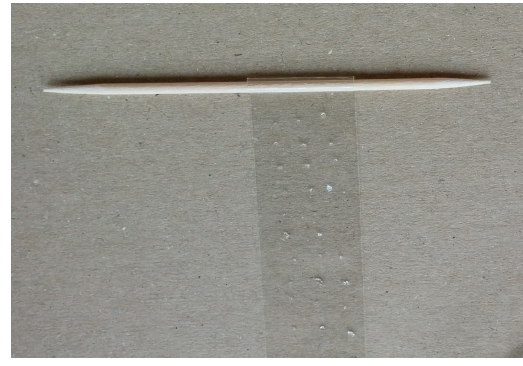

(a)

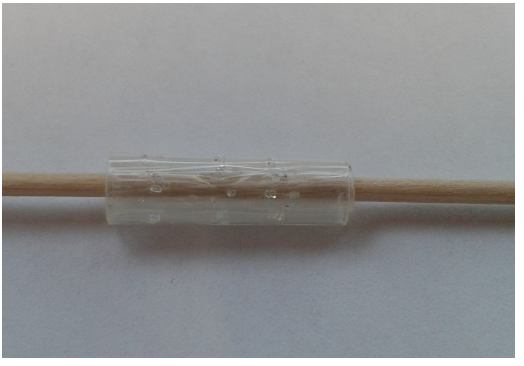

(b)

Figure 3: $\mu \mathrm{CT}$ preparation procedure. (a) Crystals are placed on an adhesive tape that is rolled around a toothpick. (b) Final tape with crystals.

and imaged under a microscope. The crystals that were judged to be aggregates were then prepared for imaging by microcomputed tomography $(\mu \mathrm{CT})$. This is the "Aggregates" preparation procedure referred to in Table 1. As it was observed that some crystals fell apart during preparation, both single crystals and aggregates from the $\mathrm{M}$ experiments were prepared. This ensured that the crystals are moved with tweezers only once, which decreased the probability of aggregates breaking apart. This is referred to as the preparation procedure "All". Approximately half of the sampled crystals from experiment $\mathrm{R}$ and a tenth of the samples from $\mathrm{M}$ experiments were prepared for imaging.

The $\mu \mathrm{CT}$ preparation procedure described here represents an improvement on the one we presented previously [20]. Adhesive tape was rolled around a toothpick, and the crystals to be imaged were placed on the tape in rows of three, thus ensuring sufficient separation between the particles. The tape was then rolled in further around the toothpick, which enabled this to be done in a controlled manner such that particle movement was minimized. The preparation is illustrated in Figure 3 .

The toothpick was then placed in a screw clamp holder and imaged in a ZEISS X-Radia Versa XRM $500 \mu \mathrm{CT}$ machine. The measurement was performed using the parameters shown in Table 2, The proprietary software XRM Reconstructor was used for CT reconstruction. The resulting volume was cropped 


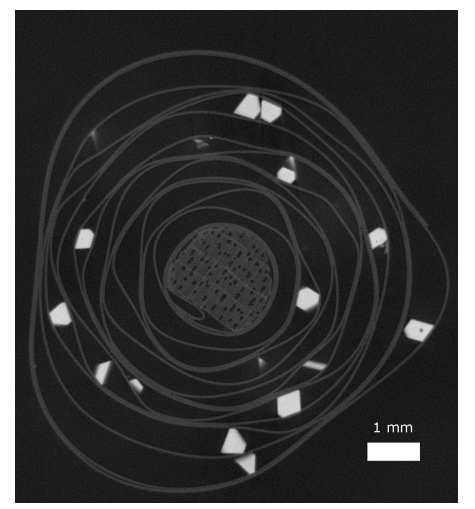

Figure 4: One slice from experiment M5, in which the onion-like adhesive tape layers around the porous-looking toothpick can be seen. Crystals that are present in the slice are shown in white.

and a .tif stack containing 2D slice images was exported. One of the slices from experiment M5 can be seen in Figure 4. Because the crystals are much brighter than the toothpick and the adhesive tape, they can easily be segmented. A binarization using the Otsu threshold [30] [p. 515-516] was performed for all experiments except for M10, for which the threshold was found manually by observing the effect on different slices using the MAVI software [31].

\subsection{Simulated Data}

\subsubsection{Simulated Single Crystals}

In order to assess the accuracy of the algorithm for the given resolution, we created crystals and their corresponding 3D images by simulation. First, 100 single octahedral crystals with a unit face distance were simulated. Their orientation was determined by a rotation matrix computed from the Z-Y-Z convention of Euler angles $(\alpha, \beta, \gamma)$. The angles $\alpha$ and $\gamma$ were sampled uniformly from 0 to $2 \pi$, whereas the cosine of the angle beta was uniformly sampled between -1 and 1 , which ensured that the resulting rotations were uniform 32. Five different samples were obtained by setting the volume-equivalent sphere diameter of the octahedra to values from $200 \mu m$ to $600 \mu m$ with an increment of $100 \mu \mathrm{m}$. We then simulated 3D images of these octahedra using 


\begin{tabular}{|c|c|c|}
\hline Parameter / Experiment(s) & $\mathrm{R}$ & $\mathrm{M}$ \\
\hline \hline Tube Voltage & $80 \mathrm{kV}$ & $80 \mathrm{kV}$ \\
\hline Power & $6 \mathrm{~W}$ & $6 \mathrm{~W}$ \\
\hline Exposure Time & $5 \mathrm{~s}$ & $5 \mathrm{~s}$ \\
\hline Magnification objective & $0.39 \mathrm{x}$ & $0.39 \mathrm{x}$ \\
\hline Source-to-sample distance & $25 \mathrm{~mm}$ & $30 \mathrm{~mm}$ \\
\hline Detector-to-sample distance & $100 \mathrm{~mm}$ & $100 \mathrm{~mm}$ \\
\hline No. projections & 1601 & 1601 \\
\hline
\end{tabular}

Table 2: Parameters of the $\mu \mathrm{CT}$ measurement.

the resolution of $7.85 \mu \mathrm{m}$ per voxel, as in the M experiments. The details of how a 3D image with a given resolution is obtained from the octahedron with given vertices can be found in our previous work [20].

\subsubsection{Simulated Crystal Aggregates}

To further show that the algorithm is capable of measuring the DAD, we simulated a sample of 100 crystal aggregates using the approach we presented previously [20]. The aggregates consisted of two to five primary particles. Each particle had a random orientation, where the rotation matrix was sampled uniformly as explained in Section 3.3.1 The primary particle face distance $h$ was sampled from a normal distribution with a mean of $250 \mu \mathrm{m}$ and a standard deviation of $30 \mu \mathrm{m}$. The particles were first brought into contact where they lightly touch. Next, they were grown by increasing the face distance of each particle by $50 \mu \mathrm{m}$. The 3D images were simulated using a voxel length of 15 $\mu m$.

\section{Aggregate Segmentation and Shape Identification}

The presented algorithms were implemented in MATLAB 2015b, with MATLAB 2014a used for visualization [33]. They use our previous framework regarding convex geometry [25, 34, image processing of $\mu \mathrm{CT}$ data [16, 20] the cdd 
library [35] and the Marching Cubes rendering algorithm [36]. We considered octahedral potash alum crystals with 8 faces, as opposed to the previously used 26 faces [16, 20]. This is justified by the shapes observed in the experiments and is less likely to lead to errors since the non-existent small non-octahedral faces would not be identified by a Hough transform.

\subsection{Aggregate Isolation}

Individual crystals were isolated from the $\mu \mathrm{CT}$ image of the sample using the connected-component labeling procedure with a 26-8 neighborhood in the MAVI software [31. Because of the improved sample preparation procedure, no further steps were necessary to ensure that crystals were indeed in contact. This is in contrast to our previous work where additional measures had to be taken (see [20, Section 3.2.2). Separated crystals were stored as individual 3D images and discarded if their volume equivalent diameters were smaller than $200 \mu \mathrm{m}$, which was the mesh size of the finer sieve.

As one aggregate from experiment $R$ was too large to enable processing with the given amount of RAM, it was down-sampled by halving it in each dimension using MAVI. This was compensated for by considering the resolution, measured by voxel length in $\mu m$, to be twice as large for this crystal.

\subsection{Aggregate Segmentation}

The algorithm for segmenting an aggregate into primary particles was presented in our previous work [20]. It is summarized briefly here and illustrated in Figure 5 for one example crystal. The algorithm considers a volume point cloud consisting of foreground voxel center coordinates, and a surface point cloud containing coordinates of voxels on the aggregate surface. The first step is finding concavity points that are located at the interface between two primary particles [37, 38. A concavity expansion procedure [39] follows, which involves deleting voxels in the concavity direction. This ensures separation near concavity points when applying the distance-transform-based watershed transform [40]. The obtained image is used to impose minima for the distance-transform-based 


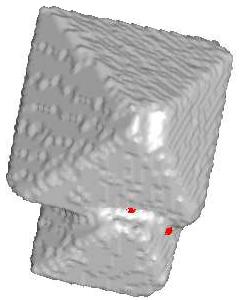

(a)

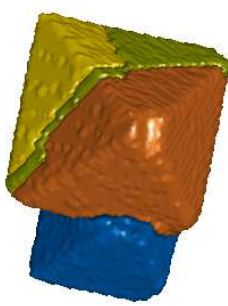

(e)

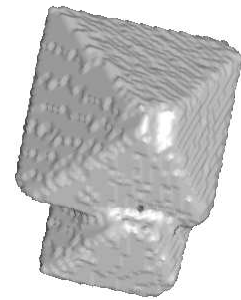

(b)

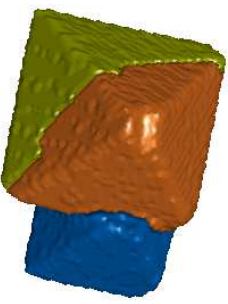

(f)

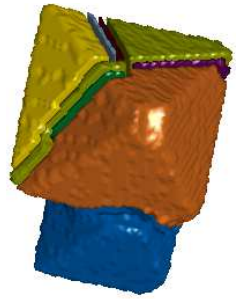

(c)

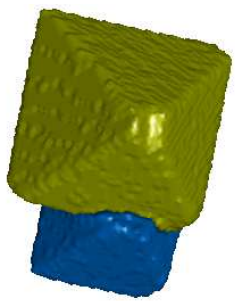

$(\mathrm{g})$

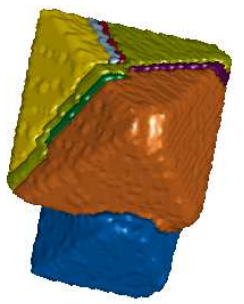

(d)

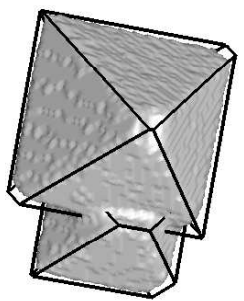

(h)

Figure 5: Segmentation example. (a) Concavity points, marked as red circles, (b) concavity expansion results in thin holes through the crystal, (c) distance-transform based watershed segmentation, resulting in watershed regions and separation watershed voxels, (d) watershed voxels are reassigned to fill the holes, (e) concatenation of small and large regions, (f) iteration 1 of concatenation of large regions, (g) final segmentation after iteration 2 of concatenation of large regions, (h) shape identification.

watershed transform of the original image. This procedure usually results in over-segmentation and the obtained watershed regions are iteratively concatenated. First, the small regions are concatenated with large ones in order not to introduce further constraints [41]. The remaining regions are concatenated [42] while considering concavity points as indicators of correct segmentation.

The previously introduced filtering step, as described in Section 3.3. of [20], is omitted. In the case considered here, where particles may be very loosely connected, the described filtering can lead to the removal of one of the particles. Furthermore, filtering is no longer necessary because the surface-roughnessinduced segmentation inaccuracies will be corrected by user interaction, as explained below. 


\subsubsection{User Interaction}

As discussed in 20, the segmentation can sometimes appear to be incorrect. We here introduce two user-interaction steps in order to resolve this issue. First, the user may remove some of the existing concavity points or add new ones if not satisfied with the automatic identification. Afterward, the segmentation steps (b)-(g) described in Figure $5 \mathrm{~h}$ are performed. The results are then displayed to the user, as illustrated in Figure 6. Here the user may decide to concatenate the obtained regions or to add new regions by clicking on some point in the point cloud, as illustrated in Figure 6. If a region is added, the program determines the region to which the selected point belonged to after the steps of watershed segmentation and watershed voxel reassignment. This region is introduced as a new region. Furthermore, if any region is split into more than one connected component by this procedure, the resulting connected components are also added as new regions. The user may repeat these steps until the result is satisfactory, at which point the segmentation information is stored stored and the algorithm proceeds with the next crystal. Even though this user interaction may introduce some non-reproducibility it should be noted that most cases in which this procedure was applied were as obvious as that shown in 6 .

In experiment M10, we observed five particles whose shape was not consistent with single or agglomerated potash alum crystals because of either breakage or other artifacts, as illustrated in Figure 7. These crystals were excluded from further consideration.

\subsection{Shape Identification}

After segmenting all aggregates into primary particles, particle shapes are identified. For each primary particle, this is based on its surface point cloud. These are the coordinates of the aggregate surface points that belong to the considered primary particle [20].

We modify the shape identification procedures presented in [20, 16] to allow asymmetrical crystals, as discussed below. In order to explain the modifications, 

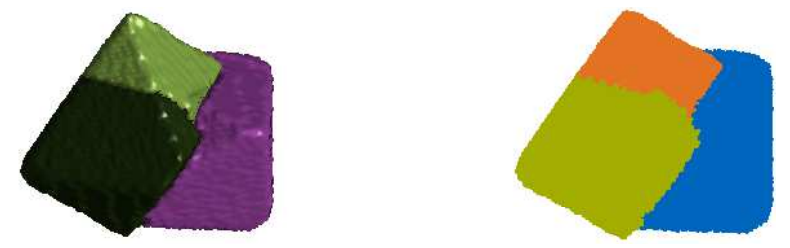

(a)
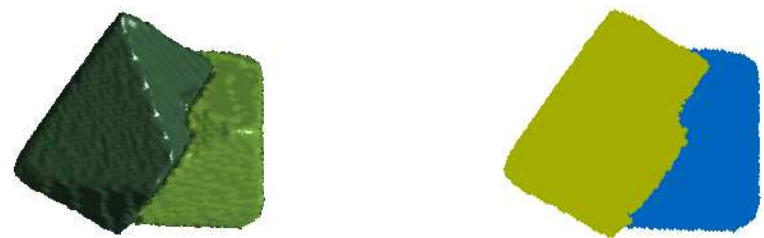

(b)

Figure 6: Region manipulation interface. The rendered crystal regions (left) as well as the volume point cloud (right) are displayed for the user. The figures are rendered in different colors to eliminate contrast issues. The user can rotate the figures and click on points in the point cloud in order to concatenate or add regions. In the presented case, the user would click on the orange and green regions in the right-hand image of (a). These would be concatenated to produce (b), whereupon the user would accept the segmentation.

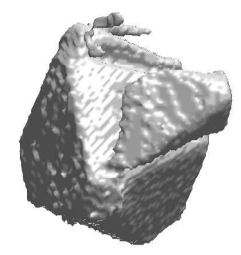

(a)

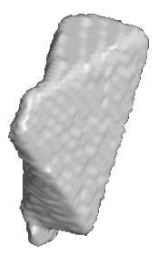

(b)

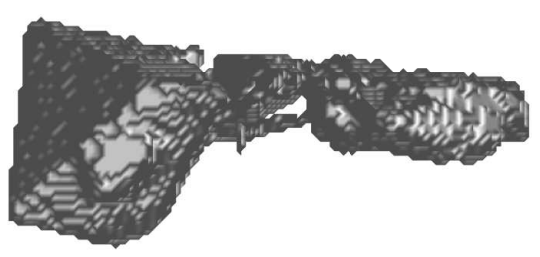

(c)

Figure 7: Some of the crystals from experiment M10 that were ignored because they did not correspond to single or agglomerated crystals. 
we first summarize the main steps from [16] and [20].

A Hough transform [43, 44] is performed in order to find crystal faces. This is a classical image processing tool where the space is discretized into directions $\mathbf{n}_{i}$ and distances $\rho_{j}$ from the primary-particle middle point, obtained as the middle point of its surface point cloud. The resulting direction-distance pairs $\left(\mathbf{n}_{i}, \rho_{j}\right)$ represent the Hough transform bins $b_{i, j}$. Each bin is then filled with surface points $\mathrm{x}$ that satisfy the following equation [16]:

$$
\rho_{j}-\frac{\Delta \rho}{2} \leq\left\langle\mathbf{n}_{i}, \mathbf{x}\right\rangle<\rho_{j}+\frac{\Delta \rho}{2}
$$

where $\Delta \rho$ is the distance grid displacement. Finally, a grid containing the maximal number of points per bin $\tilde{b}_{i}$ in each direction $\mathbf{n}_{i}$ is formed and the corresponding distance $\rho_{j}$ is stored. Face distance directions are then obtained by a peak search operation over this grid and are gathered in the matrix $\mathbf{A}_{\mathrm{F}}$.

As some crystal faces are either very small or not visible on the crystal surface, their face normals do not appear in the matrix $\mathbf{A}_{\mathrm{F}}$. Therefore, a matching between the found face normals $\mathbf{A}_{\mathrm{F}}$ and the face normals in the crystal model, denoted by $\mathbf{A}$, is performed [16]. Additionally, a rotation matrix $\mathbf{R}$ that rotates the shape model into the identified shape is determined [16].

It remains to find the face distances from the middle point for each rotated model face normal $\mathbf{R} \cdot \mathbf{a}_{i}$. Here we adapt the approach from [16]. If a match in $\mathbf{A}_{\mathbf{F}}$ exists for this normal, the face distance is obtained as the distance $\rho_{j}$ corresponding to the Hough transform bin $b_{i, j}$ with the highest point count in the direction $\mathbf{n}_{i}$ closest to $\mathbf{R} \cdot \mathbf{a}_{i}[16]$. Otherwise, we consider the points within each bin $b_{i, j}$ in the direction $\mathbf{n}_{i}$. The points are then projected to the plane defined by $\left(\mathbf{n}_{i}, \rho_{j}\right)$ and the convex hull of the projections is calculated. The face distance is obtained as the distance $\rho_{j}$ that leads to the highest point number density:

$$
r_{j}=\frac{b_{i, j}}{A_{i, j}}
$$

where $A_{i, j}$ is the area of the obtained convex hull.

The main modification to our previous approaches [20, 16, is to ignore the symmetry conditions and accept the obtained asymmetrical shape. We observed 


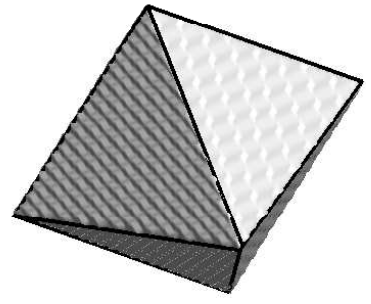

(a)

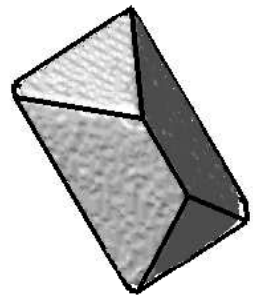

(b)

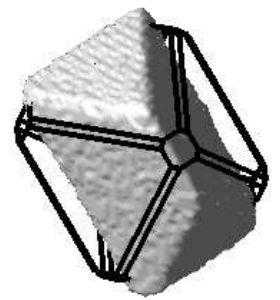

(c)

Figure 8: A simulated symmetrical crystal (a) and an observed asymmetrical crystal from experiment M3 (b,c). The shape identification in (b) was performed using the algorithm presented in this work. The use of our previously published algorithm, based on the symmetrical model, results in an inadequate shape, as seen in (c). Note that the previously published algorithm used a crystal model with 26 faces.

highly asymmetrical crystals in the experimental data presented here. The issues regarding using a symmetrical model on an aggregate of asymmetrical crystals were already discussed in our previous work 20. In Figure 8, the difference between (a) symmetrical and (b, c) asymmetrical crystals is highlighted. There is a large difference in shape identification using (b) the modified algorithm with the asymmetrical shape model and (c) the algorithm based on symmetry [20].

Two post-processing steps are introduced to resolve arising issues such as nucleation.

Small Crystals. We exclude primary particles whose volume-equivalent sphere diameter is smaller than $200 \mu \mathrm{m}$, which is the mesh size of the finer sieve used to obtain the seed crystals. In the performed experiments, especially the M batches, we observed many smaller crystals attached to larger ones. The small particles could have been formed by nucleation toward the end of the experiment. However, we also observed them at the beginning of the M experiments. We assume that these are particles that were attached to the larger ones during sieving, stayed in the sieve with a larger pore size, and were then detached when dispersed in saturated solution. This is more prominent in Figure 2b, showing one image from experiment M3, than in Figure 2a, where one image from the 
initial dispersion of the experiment $\mathrm{R}$ is presented. The used resolution of the $\mu \mathrm{CT}$ system is not high enough to enable shape identification of small particles in each case. It is important to note that a particle as a whole may be larger than $200 \mu \mathrm{m}$, but it is still excluded because its visible (segmented) part is smaller than this threshold, whereas the remaining parts may overlap with other primary particles.

Deleting Faulty Regions. The shape identification may also fail for some larger particles, although this rarely happens. The primary particles, or regions, where no shape identification was possible were, therefore, deleted from the aggregate point cloud [20].

Note that these post-processing steps differ from those introduced in [20]. The steps from that work assumed that the identified shapes were symmetrical and attempted to correct the faulty segmentation that is here resolved by user interaction.

\subsection{Goodness of Fit}

The shapes of some crystals was not identified, mostly because of their size. Furthermore, if segmentation issues could not be resolved, the identified shape may be inadequate. In order to discriminate between these cases and ensure that only well-identified crystals are used to obtain the DAD, the quality of fit should be determined. Thus, we define three different measures, as in our previous work [20], except that the mean crystal face distance must be additionally determined because no symmetry conditions were imposed. The first measure is the mean quadratic distance deviation [20]:

$$
q_{\text {all }}=\frac{\sqrt{\frac{1}{N_{\text {surf,agg }}} \sum_{j=1}^{N_{\text {surf }, \text { agg }}} \min _{i}\left\|\mathbf{p}_{j}-\mathbf{p}_{j, \text { proj }, i}\right\|^{2}}}{\max _{i} h_{i, \text { mean }}}
$$

Here, $N_{\text {surf,agg }}$ aggregate surface points $\mathbf{p}_{j}$ are projected to the identified primary particles $i$, as explained in our previous work [20, 16. The value is scaled

by the maximal $h_{i, \text { mean }}$, which is the mean identified face distance for the $i$-th primary particle of the aggregate. 
We further exclude points belonging to particles for which no shape fit could be obtained, so that only $N_{\text {surf,fit }}$ points remain. This results in the following quality measure [20]:

$$
q_{\mathrm{fit}}=\frac{\sqrt{\frac{1}{N_{\text {surf fit }}} \sum_{j=1}^{N_{\text {surf fit }}} \min _{i}\left\|\mathbf{p}_{j}-\mathbf{p}_{j, \operatorname{proj}, i}\right\|^{2}}}{\max _{i} h_{i, \text { mean }}}
$$

Finally, the third quality measure is the same as in our previous work and considers the volume deviation of the fit to the voxels of the point cloud [20]:

$$
q_{v}=\frac{N_{\text {outside }}}{N_{\text {voxels }}}+\frac{V_{\text {agg }}-N_{\text {inside }}}{N_{\text {voxels }}} .
$$

Here, $N_{\text {outside }}$ and $N_{\text {inside }}$ are the numbers of voxels outside and inside the identified shapes, $N_{\text {voxels }}$ is the number of voxels in the aggregate image approximating its volume, and $V_{\text {agg }}$ is the volume of the aggregate computed using the volumes of the fitted polytopes under consideration of their overlaps [20]. Note that the voxel is considered to be inside the shape if the point represented by its coordinates is inside the shape.

A crystal (aggregate or single particle) is considered to have a "good" shape fit if a fit is possible for all identified primary particles and $q_{\text {all }}<0.15$ and $q_{v}<0.2$. An aggregate with "missing" primary particles is declared if $q_{\mathrm{fit}}<0.15$ and there exist primary particles for which no shape identification is possible. Otherwise, the shape fit for the aggregate is declared as "bad". Examples of these classifications can be seen in Figure 9

This classification is created in order to exclude "bad" aggregates from consideration when computing the DAD. However, only one crystal was classified as "bad" for the four experimental samples and one simulated sample that were considered, as seen in Table 3. As that particle was a single crystal, the classification has no influence on the final DAD, but serves as a quality check for the shape identification.

\subsection{Disorientation Angle Distribution}

The DAD is used in material science to describe the properties of a material in terms of the orientation of the crystallites. Mackenzie and Thomson [45] ap- 


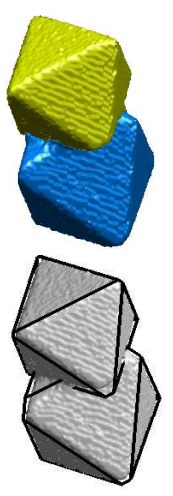

(a)

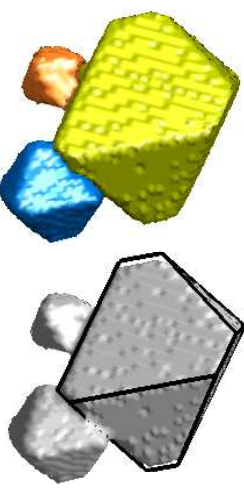

(b)

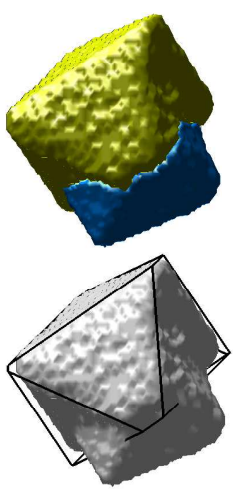

(c)

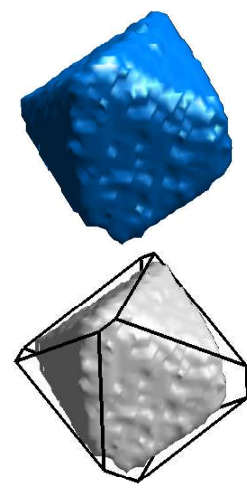

(d)

Figure 9: Examples of crystals according to their goodness of fit. The upper row shows the segmentation into primary particles. In the lower row, identified shapes are marked in black. Aggregate (a) was declared to have a "good" fit. Aggregate (b) has "missing" primary particles, where the orange and blue particles were ignored because of their size. Crystal (c) has "missing" primary particles, where the algorithm failed to provide a shape identification for the blue particle. Single crystal (d) was judged to have a "bad" fit.

\begin{tabular}{|c||c|c|c|}
\hline Sample/Type & Good & Missing & Bad \\
\hline \hline R & 46 & 15 & 0 \\
\hline M3 & 101 & 31 & 1 \\
\hline M5 & 104 & 55 & 0 \\
\hline M10 & 101 & 48 & 0 \\
\hline
\end{tabular}

Table 3: Number of "good", "missing" and "bad" crystals in each sample. 
proximated the DAD for randomly oriented cubic crystals using a Monte Carlo simulation. This was followed quickly by the derivation of an analytical solution by Handscomb 23] using the quaternion representation of the rotations. This solution is given in the appendix. Mackenzie 24] published the same solution using another approach at about the same time. In the following, we give the definition of the disorientation angle and apply the approach of Mackenzie and Thomson 45] to find the DAD of the imaged potash alum crystals.

\subsubsection{Disorientation Angle Definition}

Consider a cube A that has some reference orientation, and a cube B obtained by rotating A using some rotation matrix $\mathbf{R}$. The misorientation angle of the cubes $\mathrm{A}$ and $\mathrm{B}$ is the angle corresponding to this rotation in the axis-angle representation [46]. However, the same orientation between the crystals is obtained if one of the 24 symmetric rotations $\mathbf{R}_{i}$ is applied to cube B. Therefore, each rotation combination $\mathbf{R}_{i} \mathbf{R}$ results in one misorientation angle $\Theta_{i}$. The smallest of these angles is called the disorientation angle $\Theta$ and is obtained as in 45

$$
\Theta=\min _{i} \Theta_{i},
$$

where the misorientation angles $\Theta_{i}$ can be computed from the rotation matrices using [4]:

$$
\operatorname{tr}\left(\mathbf{R}_{i} \mathbf{R}\right)=1+2 \cos \left(\Theta_{i}\right)
$$

where tr is the trace operator that sums the diagonal matrix elements.

As the octahedron is the dual body of the cube, the same considerations can be applied to the potash alum crystals. The reference orientations of a cube and an octahedron are illustrated in Figure 10.

The 24 symmetry operations $\mathbf{R}_{i}$ are defined for these reference orientations as 24]:

- Identity matrix

- Rotations for $90^{\circ}, 180^{\circ}$, and $270^{\circ}$ around the coordinate system axes $\mathbf{x}$, $\mathbf{y}$, and $\mathbf{z}$, leading to 9 rotation matrices 


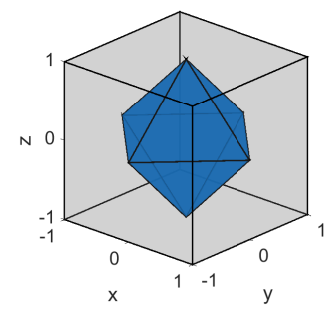

Figure 10: Reference orientation of cube and octahedron.

- Rotations for $180^{\circ}$ around axes parallel to the face diagonals of the reference cube, leading to 6 rotation matrices

- Rotations for $120^{\circ}$ and $240^{\circ}$ around the four reference cube diagonals, leading to 8 rotation matrices

Let us now consider two identified crystals in contact whose vertices are $\mathbf{V}_{\mathrm{A}}$ and $\mathbf{V}_{\mathrm{B}}$. In order to determine the disorientation angle, each crystal is translated such that its center is at the origin. The corresponding H-representation is determined leading to the matrices of face normals, $\mathbf{A}_{\mathrm{A}}$ and $\mathbf{A}_{\mathrm{B}}$. Here, the obtained matrices have to contain face normals in the same order as defined for the reference octahedron $\mathbf{A}_{\mathrm{r}}$, and are permuted to fulfill this condition if this was previously not the case. A unit face distance is assigned to both octahedra to eliminate the influence of crystal size. The rotation matrix $\mathbf{R}_{1}$ that rotates the crystal $\mathbf{A}_{\mathrm{A}}$ into the reference position $\mathbf{A}_{\mathrm{r}}$ is now determined:

$$
\mathbf{R}_{1}=\left(\mathbf{A}_{A_{1: 3}}^{-1} \cdot \mathbf{A}_{\mathrm{r}_{1: 3}}\right)^{T} .
$$

Here, $\mathbf{A}_{A_{1: 3}}$ denotes that only the first three rows of the matrix are considered when determining the rotation matrix. This rotation is applied to both crystals $\mathbf{A}_{\mathrm{A}}$ and $\mathbf{A}_{\mathrm{B}}$ so that the obtained crystal $\tilde{\mathbf{A}}_{\mathrm{A}}$ now has the reference orientation, where the relative orientation between the two crystals remains unchanged:

$$
\begin{aligned}
\tilde{\mathbf{A}}_{\mathrm{A}} & =\mathbf{A}_{\mathrm{A}} \mathbf{R}_{1}^{T} \\
\tilde{\mathbf{A}}_{\mathrm{B}} & =\mathbf{A}_{\mathrm{B}} \mathbf{R}_{1}^{T}
\end{aligned}
$$




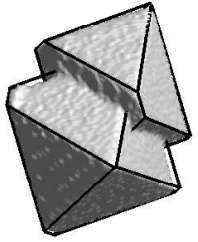

(a) $\Theta=2.4$

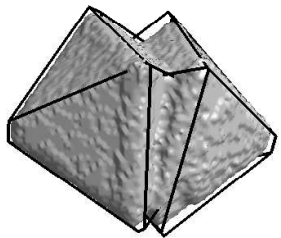

(b) $\Theta=15.3$

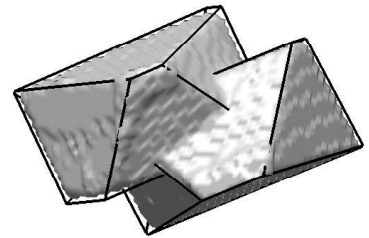

(c) $\Theta=49.7$

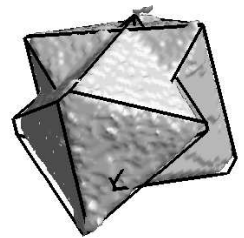

(d) $\Theta=52.3$

Figure 11: Examples of disorientation angles between some aggregates from the experiment M10.

The rotation matrix $\mathbf{R}$ that rotates the reference crystal $\tilde{\mathbf{A}}_{\mathrm{A}}$ into the crystal $\tilde{\mathbf{A}}_{\mathrm{B}}$ is obtained as

$$
\mathbf{R}=\left(\mathbf{A}_{\mathrm{r}_{1: 3}}^{-1} \cdot \tilde{\mathbf{A}}_{\mathrm{B}_{1: 3}}\right)^{T} .
$$

The disorientation angle $\Theta$ is computed as the minimal angle $\Theta_{i}$ :

$$
\Theta_{i}=\arccos \left(\frac{\operatorname{tr}\left(\mathbf{R}_{i} \mathbf{R}\right)-1}{2}\right)
$$

Different angles $\Theta_{i}$ are obtained using the 24 symmetry rotation matrices $\mathbf{R}_{i}$. These rotation matrices correspond to the symmetry operations defined above executed on the reference coordinate system rotated by $\mathbf{R}$.

It is important to note that the crystal asymmetry does not influence this procedure. The identified shapes $\mathbf{V}_{\mathrm{A}}$ and $\mathbf{V}_{\mathrm{B}}$ may not represent perfect octahedra, but their face normals in $\mathbf{A}_{\mathrm{A}}$ and $\mathbf{A}_{\mathrm{B}}$ are the octahedral face normals by the design of the shape identification algorithm. Hence, the symmetry operations for octahedra can be applied because the faces are equivalent.

Some examples of disorientation angles for aggregates of two primary particles can be seen in Figure 11 .

\subsubsection{Classification of Primary Particle Contact}

The imaged aggregates consisted of both loosely bound and grown-in primary particle pairs. Note that here pairs of primary particles are investigated instead of aggregates, as aggregates may consist of a mixture of lightly touching and grown-in pairs. We consider only the DAD of the grown-in, agglomerated 
pairs to be reliable. This is because it is not possible to determine whether the loosely bound pairs came into contact very recently within the crystallizer vessel, or were formed by particles falling next to each other on the filter paper. Additionally, the orientation between the lightly-touching particles can be changed accidentally during the preparation procedure that involves rolling the adhesive tape around the toothpick; this cannot happen for crystals that have grown inside each other. Thus, it is necessary to classify the contact between the pairs of primary particles.

Some cases of primary particle contact can be ambiguous. For example, if a small crystal lies on the surface of a large crystal, given the crystal asymmetry, it is not possible to determine whether the crystals are grown in or only lightly touching. Furthermore, the classification cannot be performed automatically because the segmentation and fitting may be erroneous. For this reason, the classification is performed by experts with knowledge in the field of imaging/crystallization, as is typically done when creating a machine classifier [14]. The expert is shown an aggregate, together with the identified shapes of the two primary particles whose contact is to be classified. The expert classifies the pair as "grown in", "lightly touching" or "none", where "none" means that the particles are not in contact. In order to do this, the expert may rotate the rendered particles and change the light settings. We asked three different experts to perform the classification in order to avoid bias in ambiguous cases. The instructions for the experts contained the following descriptions of the three contact classes:

- "Lightly touching" are all pairs of particles that could have been brought into contact without growth, so by simply placing them next to each other or onto each other. They can be separated without harming one of them.

- "Grown in" are crystals where one is grown strongly into the other and they cannot be separated without harm.

- "None" are crystals that are not in contact. 


\begin{tabular}{|c||c|c|c|c||c|c|c|c||c|c|c|c||c|c|c|c|}
\hline \multicolumn{1}{|c||}{} & \multicolumn{9}{c||}{ R } & \multicolumn{4}{c||}{ M3 } & \multicolumn{4}{c||}{ M5 } & \multicolumn{4}{c|}{ M10 } \\
\hline \hline Expert & H & M & T & all & H & M & T & all & H & M & T & all & H & M & T & all \\
\hline grown-in & 40 & 40 & 40 & 40 & 35 & 33 & 34 & 33 & 30 & 32 & 29 & 28 & 29 & 26 & 26 & 26 \\
\hline touching & 6 & 7 & 6 & 5 & 40 & 43 & 41 & 40 & 53 & 50 & 53 & 46 & 34 & 35 & 35 & 32 \\
\hline none & 5 & 4 & 5 & 4 & 15 & 14 & 15 & 14 & 31 & 32 & 32 & 27 & 21 & 23 & 23 & 21 \\
\hline
\end{tabular}

Table 4: Number of primary particle pairs from experiments R, M3, M5, and M10 that were classified as "grown-in", "lightly touching" and "none" by three different experts, denoted by $\mathrm{H}, \mathrm{M}$ and $\mathrm{T}$. Also, the number of pairs that were classified into the same class by all three experts, denoted by "all".

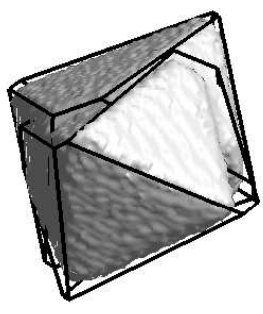

(a) Grown in

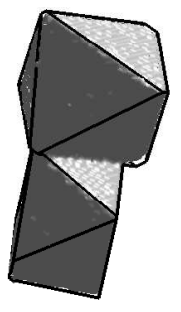

(b) Lightly touching

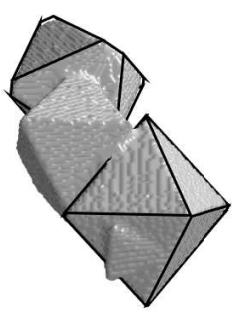

(c) None

Figure 12: Examples of contact classes.

The experts were also shown an example of each class. The examples belong to sample M5 and can be seen in Figure 12. In Figure 12c, experts considered only the framed crystals. They do not touch directly, which can be confirmed by rotating the aggregate, so the pair is classified as not touching ("none"). Table 4 shows the number of particles classified into each class by each expert. It can be seen that less than $15 \%$ of primary particle pairs were excluded from consideration by merging the opinions of the experts. Only the crystals that all three experts placed in the same category, corresponding to entries "all" in Table 4, were used for the DAD. 


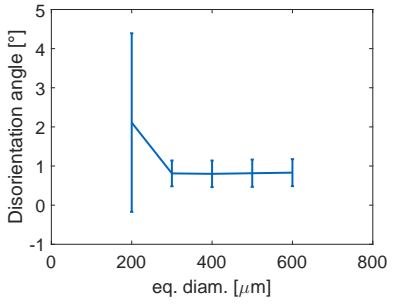

(a) Mean angle error

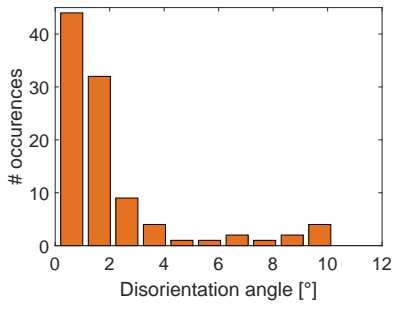

(b) eq. diam. $=200 \mu m$

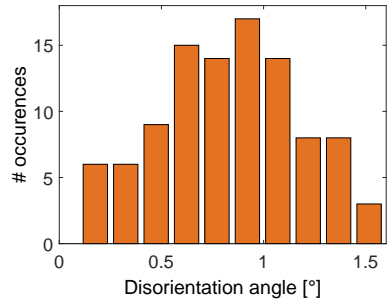

(c) eq. diam. $=600 \mu m$

Figure 13: Simulation results for single crystals. Angle error is expressed as disorientation angle between the simulated polytope and the polytope identified from the simulated image. (a) shows the dependence of the mean disorientation angle (angle error) for the sample on the simulated volume-equivalent sphere diameter. (b) and (c) show the angle error distributions for the equivalent diameters of $200 \mu \mathrm{m}$ and $600 \mu \mathrm{m}$, respectively. The 3D images were simulated using the resolution of $7.85 \mu \mathrm{m}$ per voxel length, corresponding to the resolution used for imaging the $\mathrm{M}$ experiments.

\section{Results and Discussion}

\subsection{Simulated Data}

In order to ensure that the angular information for the experimental crystals is meaningful, the introduced procedure is tested with simulated crystals with known orientation. First, images of single crystals are considered. The images were obtained using the procedure from Section 3.3.1. The accuracy of the orientation/angle estimation is assessed in relation to the crystal size. The angle error, measured as the disorientation angle between the simulated and identified polytope, is shown in Figure 13a. The error distributions for the

volume-equivalent sphere diameters of $200 \mu \mathrm{m}$ and $600 \mu \mathrm{m}$ are presented in Figures $13 \mathrm{~b}$ and $13 \mathrm{c}$

It can be seen that the mean angle error is largest at $2.11^{\circ}$ for the crystals with an equivalent diameter of $200 \mu \mathrm{m}$ and reduces to $<1^{\circ}$ for larger crystals. Furthermore, even for the equivalent diameter of $200 \mu m$, most angle errors were below $3^{\circ}$, as seen in Figure $13 \mathrm{~b}$

We further measured the DAD for the simulated sample of 100 aggregates, described in Section 3.3.2. Primary particle orientations were sampled from a 


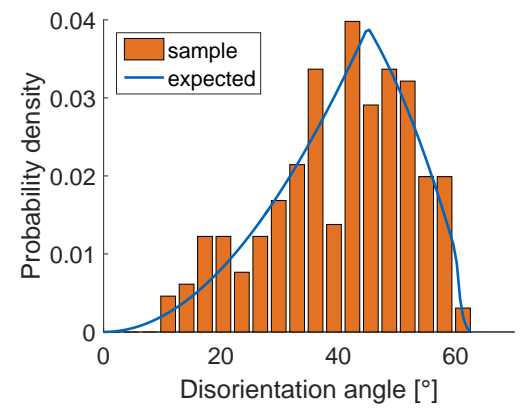

(a) Simulated polytopes

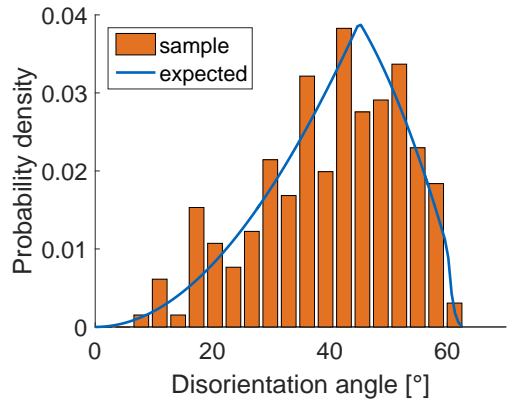

(b) Identified polytopes

Figure 14: Original DAD for the simulated polytopes (a) and the polytopes identified from the simulated 3D images (b). The data set contained 100 simulated aggregates. In both cases, 208 primary particle pairs were identified as being in contact, corresponding to both "grown-in" and "lightly touching" pairs. The blue line is the expected theoretical distribution [23, 24]

uniform distribution. First, the DAD for the simulated polytopes is computed. It is compared to the DAD computed after performing shape identification in 3D images based on these simulated polytopes. The two measured distributions are shown in Figure 14. The quality of the simulated images with respect to the resolution is in the same range as that of the simulated single crystals. When considering simulated polyotpes directly, we present the DAD for pairs of primary particles that intersect. As the correct polyotpe coordinates are known, there is no need for classification. When considering polytopes that were identified from the simulated images, an expert classified the contact type into "grown-in", "lightly touching", and "none", as described in Section 4.5. The presented DAD is for the combination of "grown-in" and "lightly touching", leading therefore to all primary particle pairs that were in contact.

It can be seen that the DADs for both the simulated and identified poyltopes follow the theoretically predicted distribution derived by Mackenzie [24] and Handscomb 23]. The agreement is good, even though there are some differences in the height of the individual bars of the two distributions. The obtained mean angle values are $40.16^{\circ}$ for the simulated polytopes and $40.18^{\circ}$ for the identified polytopes, which agree well with the theoretically expected value of $42.7^{\circ}[23$. 


\begin{tabular}{|c||c|c|c||c|c|c||c|c|}
\hline $\begin{array}{c}\text { Sample } \\
\text { / } \\
\text { Type }\end{array}$ & $\begin{array}{c}\text { single } \\
\text { all } \\
\text { fitted }\end{array}$ & $\begin{array}{c}\text { single } \\
\text { with } \\
\text { small }\end{array}$ & $\begin{array}{c}\text { single } \\
\text { with } \\
\text { failed }\end{array}$ & $\begin{array}{c}\text { agg. } \\
\text { all } \\
\text { fitted }\end{array}$ & $\begin{array}{c}\text { agg. } \\
\text { with } \\
\text { small }\end{array}$ & $\begin{array}{c}\text { agg. } \\
\text { with } \\
\text { failed }\end{array}$ & $\begin{array}{c}\text { no fit } \\
\text { due to } \\
\text { small }\end{array}$ & $\begin{array}{c}\text { no fit } \\
\text { due to } \\
\text { failed }\end{array}$ \\
\hline \hline R & 15 & 6 & 1 & 31 & 5 & 3 & 0 & 0 \\
\hline M3 & 68 & 13 & 3 & 34 & 14 & 1 & 0 & 0 \\
\hline M5 & 75 & 20 & 7 & 29 & 24 & 3 & 0 & 1 \\
\hline M10 & 74 & 24 & 5 & 27 & 13 & 6 & 0 & 0 \\
\hline
\end{tabular}

Table 5: Number of different crystal types in each sample. Columns 2-4 show single crystals, single crystals with attached small particles, and single crystals with particles for which the fitting procedure failed to produce a result. Columns 5-7 show aggregates for which a fit was possible for all primary particles, aggregates that also contained small particles, and aggregates that contained primary particles for which no fit was possible. Columns 8 and 9 show particles for which no shape fit was possible because each particle was too small and the fitting procedure failed to produce a result. Note that "single" refers to crystals for which the shape identification was possible for only one particle, whereas for "aggregates" it was possible for multiple particles.

\subsection{Batch Experimental Data}

Table 5 shows the amount of single crystals and aggregates in each sample. It can be seen that there were many single crystals and crystals with attached small particles. It is important to note that the attached small particles do not necessarily represent full crystals, and may simply be some surface roughness that the user marked as a separate particle in order not to disturb the shape fit.

Three experts performed a classification of particle contacts, as described in section 4.5.2. In order to eliminate ambiguity in the contact classification, primary particle pairs that were classified differently by different experts were discarded. The results for all four experiments are shown in Figure 15

As can be seen in the figure, all experimentally obtained distributions, presented as orange bar graphs, differ from the theoretically derived distribution 23. 24, shown as a blue line. The theoretical distribution is derived under the assumption that the orientation of each primary particle is uniformly distributed. Under such conditions, the most probable disorientation angle is $45^{\circ}$, 

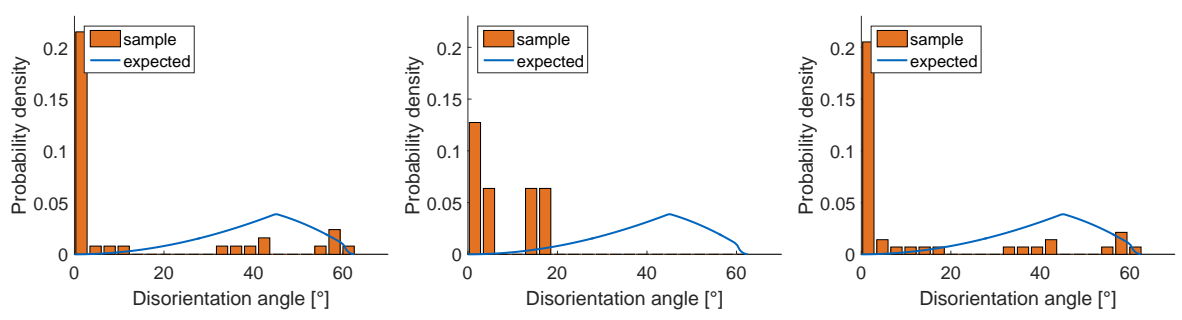

(a) R, grown in, 40 pairs

(b) R, touching, 5 pairs

(c) R, both, 45 pairs
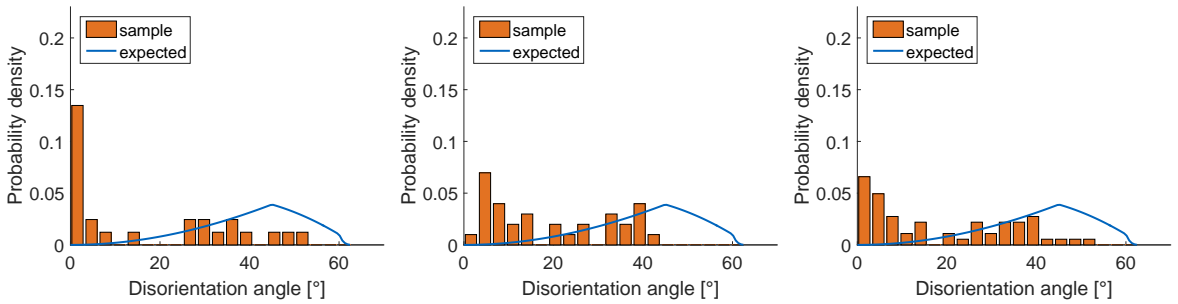

(d) M10, grown in, 26 pairs (e) M10, touching, 32 pairs

(f) M10, both, 58 pairs
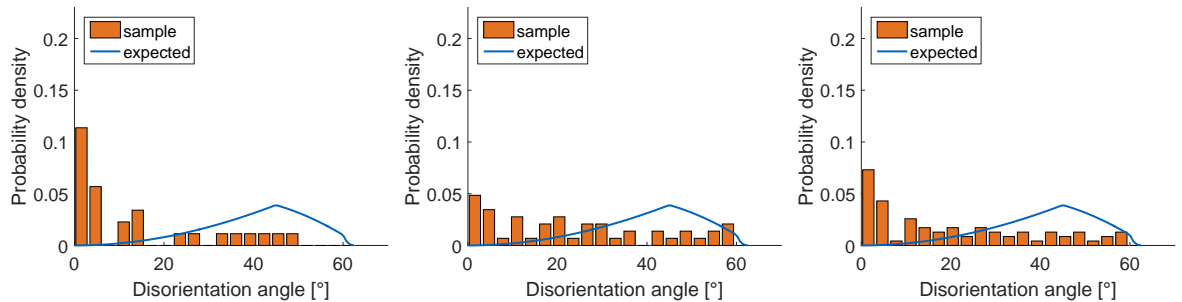

(g) M5, grown in, 28 pairs

(h) M5, touching, 46 pairs

(i) M5, both, 74 pairs
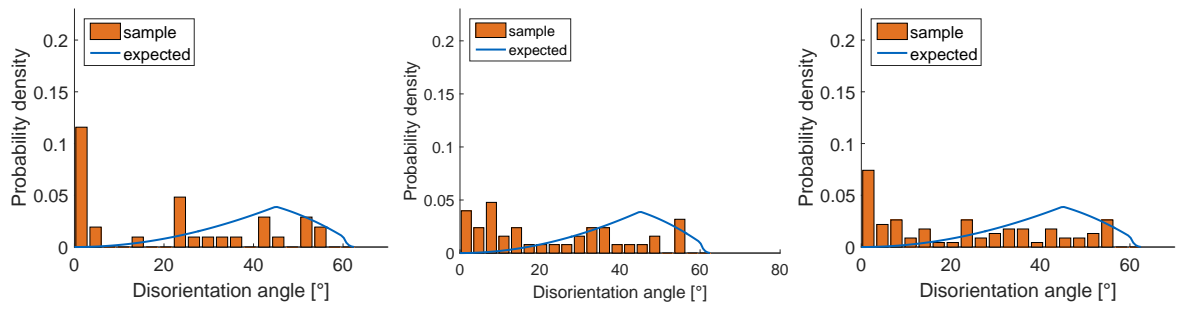

(j) M3, grown in, 33 pairs

(k) M3, touching, 40 pairs

(l) M3, both, 73 pairs

Figure 15: DAD results. Each row represents one experiment. The first column represents grown-in agglomerate pairs, the second are the pairs of lightly touching crystals, and the third column is the distribution obtained when considering grown-in agglomerate and lightly touching pairs together. The blue line represents the theoretically expected distribution [23] 24. The number of primary particle pairs included in the computation can be seen below the graphs. 
whereas a small disorientation angle-meaning that the crystals have the same orientation-is very improbable. In our experiments, however, we observed that a small disorientation angle is the most probable configuration, leading to a peak at the very left of each figure. This peak is the most prominent for grownin agglomerate pairs. As discussed in Section 4.5, in comparison with lightly touching primary particle pairs, grown-in pairs are definitely created during the batch experiment. We observed a much smaller peak in the case of lightly touching crystals, as visible in the middle column of Figure 15 . The orientation between primary particles is thus more variable in this case, as it would be expected if the particles were brought into contact during downstream processing and preparation. We think that the still unexpectedly high number of primary particles with the same orientation was caused by the fact that some of these crystals might have been created in the reactor but have not had the time to fully grow into each other. Furthermore, single potash alum crystals will always land on the filter paper in a stable position under the influence of gravity, so that they lie on one of their faces. The configurations in which one crystal lands on top of or next to another single crystal do not fulfill the assumption that their orientation is uniformly distributed and will thus lead to a deviation from the theoretically expected distribution derived under this assumption.

We observed a much stronger peak for small disorientation angles in the $\mathrm{R}$ experiment than in the $\mathrm{M}$ experiments. We think that this behavior is partially caused by the preparation procedure, which is more likely to lead to breakage of loosely bound aggregates, leading to less ambiguity as to whether the crystals are highly agglomerated or only lightly touching. Furthermore, the fact that different seed crystals were used may also have had an influence since the two manufacturers reported different impurities and the initial shape may have been different.

We did not observe a significant difference between experiments M10, M5, and M3 with different supersaturation profiles. It is possible that a difference in the distribution existed; however, the sample sizes analyzed here were not large enough to observe such a difference. This was due to a large number of single 
crystals, and also due to many pairs of lightly touching primary particles and small particles whose shapes could not be identified, as seen in Table 5 . Therefore, future studies should use more seed crystals to increase agglomeration, in which case care should be taken to avoid small particles among them. Performing the experiments at constant but highly different supersaturation levels could give a better insight into the influence of supersaturation on the particle orientation. However, high supersaturation levels could induce nucleation, which would lead back to the problem of attached small crystals that would not be resolved with the level of resolution achieved in this work.

In comparison to using a TEM, the presented $\mu \mathrm{CT}$ technique requires less effort for sample preparation and measurement, as there is no need for slicing the crystals into thin sections or manually tilting the sample [9]. More crystals could be investigated by preparing and measuring multiple adhesive tape structures, discussed in Section 3.2 .

\section{Summary, Conclusions and Outlook}

We measured the disorientation angle distribution (DAD) for pairs of primary particles in potash alum aggregates, and investigated four crystal samples grown under various conditions. In a set of three experiments (set M), we obtained different supersaturation profiles by using temperature ramps of $10 \mathrm{~K} / \mathrm{h}$, $5 \mathrm{~K} / \mathrm{h}$ and $3 \mathrm{~K} / \mathrm{h}$. The fourth experiment $(\mathrm{R})$ was performed with seed crystals from a different manufacturer, using a temperature ramp of $5 \mathrm{~K} / \mathrm{h}$ and a smaller amount of seed. The image analysis procedure was adjusted to observed artifacts caused by crystal asymmetry and small particles.

Primary particle pairs were classified as grown-in and lightly touching in order to differentiate between agglomerate pairs that were created in the reactor and those that could have been created during sampling and preparation. The DAD for grown-in agglomerate pairs showed a strong peak for small angles, so that most primary particle pairs had a very similar orientation. This is in contrast to the theoretically obtained distribution in which primary particles have 
uniformly random orientations, and leads to the conclusion that particle pairs with the same orientation are more likely to withstand the hydrodynamic forces. This behavior was most pronounced in the experiment $R$, where the peak value was larger. We assume that this was due to the different preparation procedure which involves a pre-selection of aggregates and the different seed manufacturer. Thus, theoretical predictions for uniformly distributed aggregates should not be applied to generate potash alum aggregates in simulations.

We did not observe any significant difference between the three M experiments conducted at different supersaturation levels. This contradicts the hypothesis that randomly oriented primary particles, which exhibit a lower bond strength, are more likely to survive hydrodynamic forces at higher supersaturation, where they would be bound together more quickly.

In summary, we have shown that it is possible to measure the DAD using 3D imaging and the described image processing procedure. We observed clear indications of preferential orientation for the given model compound. The same strategy could be applied to other compounds, enabling a much deeper understanding of the aggregation mechanisms during crystallization.

In order to investigate this behavior further, larger sample sizes should be used in the future to ensure more pairs of agglomerated primary particles. A better understanding of the influence of supersaturation would be obtained by experiments at constant supersaturation level with a higher difference between the chosen levels. Finally, the effect of other relevant parameters, such as mixing and crystal size, ought to be investigated. The obtained results should be coupled with single agglomerate studies, in which particle hardness for different points of contact and mutual orientations should be investigated.

\section{Acknowledgments}

The authors (TK, VW, KS, HB) are grateful for the support of Deutsche Forschungsgemeinschaft (DFG) through the Priority Program SPP 1679, Dynamic Simulation of Coupled Solids Processes, project IDs: BR-2035/9-2 and 
SU-189/6-2. We also thank Stefanie Markstein for her help during the execution of the experiments and Michael Kuhn for taking time to perform the classification of the crystal contact types. Authors FP and JS acknowledge financial support through the DFG Gottfried Wilhelm Leibniz program and the support of the TUM Institute for Advanced Study, which is funded by the German Excellence Initiative.

\section{Appendix A.}

The theoretical DAD, $p(\Theta)$ as obtained by Handscomb [23] is presented below. For an angle $\Theta \leq 45^{\circ}$ :

$$
p(\Theta)=\frac{2}{15}(1-\cos (\Theta)) .
$$

For $45^{\circ}<\Theta \leq 60^{\circ}$ :

$$
p(\Theta)=\frac{2}{15}(1-\cos (\Theta))\left(3(\sqrt{2}-1) \cot \left(\frac{1}{2} \Theta\right)-2\right) .
$$

For $60^{\circ}<\Theta \leq 60.6^{\circ}$ :

$$
p(\Theta)=\frac{2}{15}\left(\left\{3(\sqrt{2}-1)+\frac{4}{\sqrt{3}}\right\} \sin (\Theta)-6(1-\cos (\Theta)) .\right.
$$

The largest possible angle is $\Theta_{\max }=\arccos \left(\frac{1}{4}(2 \sqrt{2}-1)\right) \approx 62.8^{\circ}$, so that for $60.6^{\circ}<\Theta \leq \Theta_{\max }$ :

$$
\begin{array}{r}
p(\Theta)=\frac{2}{15}\left[\left\{3(\sqrt{2}-1)+\frac{4}{\sqrt{3}}\right\} \sin (\Theta)-6(1-\cos (\Theta))\right] \\
+\frac{8}{5 \pi}(1-\cos (\Theta))\left\{\arccos \left(\frac{\cot ^{2}\left(\frac{1}{2} \Theta\right)}{3+2 \sqrt{2}-\cot ^{2}\left(\frac{1}{2} \Theta\right)}\right)\right. \\
\left.+\frac{1}{2} \arccos \left(\frac{\cot ^{2}\left(\frac{1}{2} \Theta\right)-2 \sqrt{2}}{3-\cot ^{2}\left(\frac{1}{2} \Theta\right)}\right)\right\} \\
-\frac{8}{5 \pi} \sin (\Theta)\left\{2(\sqrt{2}-1) \arccos \left(\frac{(\sqrt{2}-1) \cot \left(\frac{1}{2} \Theta\right)}{\left(1-(\sqrt{2}-1)^{2} \cot ^{2}\left(\frac{1}{2} \Theta\right)\right)^{\frac{1}{2}}}\right)\right. \\
+\frac{1}{\sqrt{3}} \arccos \left(\frac{\left(\sqrt{(2)-1)^{2} \cot \left(\frac{1}{2} \Theta\right)}\right.}{\left.\left(3-\cot ^{2}\left(\frac{1}{2} \Theta\right)\right)^{\frac{1}{2}}\right)}\right\} .
\end{array}
$$




\section{References}

[1] J. W. Mullin, Crystallization, 3rd Edition, Butterworth-Heinemann, 2001.

[2] H. S. Mumtaz, M. J. Hounslow, N. A. Seaton, W. R. Paterson, Orthokinetic aggregation during precipitation: A computational model for calcium oxalate monohydrate, Chem. Eng. Res. Des. 75 (2) (1997) 152 - 159.

[3] M. J. Hounslow, H. S. Mumtaz, A. P. Collier, J. P. Barrick, A. S. Bramley, A micro-mechanical model for the rate of aggregation during precipitation from solution, Chem. Eng. Sci. 56 (7) (2001) $2543-2552$.

[4] M. J. Hounslow, E. J. W. Wynn, M. Kubo, K. Pitt, Aggregation of growing crystals in suspension: I. Mumtaz revisited, Chem. Eng. Sci. 101 (2013) 731-743.

[5] D. R. Ochsenbein, T. Vetter, M. Morari, M. Mazzotti, Agglomeration of needle-like crystals in suspension. ii. modeling, Cryst. Growth. Des. 15 (9) (2015) 4296-4310.

[6] H. Briesen, Hierarchical characterization of aggregates for Monte Carlo simulations, AIChE J 52 (7) (2006) 2436-2446.

[7] H. Briesen, Aggregate Structure Evolution for Size-Dependent Aggregation by Means of Monte Carlo Simulations, KONA Powder Part. J. 25 (2007) 180-189.

[8] V. K. Ivanov, P. P. Fedorov, A. Y. Baranchikov, V. V. Osiko, Oriented attachment of particles: 100 years of investigations of non-classical crystal growth, Russ. Chem. Rev. 83 (12) (2014) 1204-1222.

[9] A. P. Collier, C. J. D. Hetherington, M. J. Hounslow, High resolution imaging of crystalline agglomerates, Chem. Eng. Res. Des. 74 (A7) (1996) 759764, 13th Symposium on Industrial Crystallization, Toulouse, France, SEP 16-19, 1996. 
[10] A. P. Collier, C. J. D. Hetherington, M. J. Hounslow, Alignment mechanisms between particles in crystalline aggregates, J. Cryst. Growth 208 (14) (2000) $513-519$.

[11] N. Faria, M. N. Pons, S. Feyo de Azevedo, F. A. Rocha, H. Vivier, Quantification of the morphology of sucrose crystals by image analysis, Powder Technol. 133 (1-3) (2003) 54-67.

[12] A. Ferreira, N. Faria, F. Rocha, J. A. Teixeira, Using an Online Image Analysis Technique to Characterize Sucrose Crystal Morphology during a Crystallization Run, Ind. Eng. Chem. Res. 50 (11) (2011) 6990-7002.

[13] L.-M. Terdenge, S. Heisel, G. Schembecker, K. Wohlgemuth, Agglomeration degree distribution as quality criterion to evaluate crystalline products, Chem. Eng. Sci. 133 (2015) $157-169$.

[14] D. R. Ochsenbein, T. Vetter, S. Schorsch, M. Morari, M. Mazzotti, Agglomeration of needle-like crystals in suspension: I. measurements, Cryst. Growth. Des. 15 (4) (2015) 1923-1933.

[15] M. R. Singh, J. Chakraborty, N. Nere, H.-H. Tung, S. Bordawekar, D. Ramkrishna, Image-Analysis-Based Method for 3D Crystal Morphology Measurement and Polymorph Identification Using Confocal Microscopy, Cryst. Growth Des. 12 (7) (2012) 3735-3748.

[16] T. Kovačević, A. Reinhold, H. Briesen, Identifying faceted crystal shape from three-dimensional tomography data, Cryst. Growth Des. 14 (4) (2014) $1666-1675$.

[17] J.-H. Hours, S. Schorsch, C. N. Jones, Parametric Polytope Reconstruction, an Application to Crystal Shape Estimation, IEEE Trans. Image Process. 23 (10) (2014) 4474-4485.

[18] S. Schorsch, J.-H. Hours, T. Vetter, M. Mazzotti, C. N. Jones, An optimization-based approach to extract faceted crystal shapes from stereoscopic images, Comput. Chem. Eng. 75 (2015) 171-183. 
[19] C. Y. Ma, J. J. Liu, X. Z. Wang, Stereo imaging of crystal growth, AIChE J 62 (1) (2016) 18-25.

[20] T. Kovačević, J. Schock, F. Pfeiffer, H. Briesen, Shape identification of primary particles in potash alum aggregates using three-dimensional tomography data, Cryst. Growth Des. 16 (5) (2016) 2685-2699.

[21] M. Brunsteiner, A. G. Jones, F. Pratola, S. L. Price, S. J. R. Simons, Toward a molecular understanding of crystal agglomeration, Cryst. Growth Des. 5 (1) (2005) 3-16.

[22] H.-J. Bunge, Texture Analysis in Material Science, Butterworths, 1982.

[23] D. C. Handscomb, On the random disorientation of two cubes, Canad. J. Math. 10 (1958) 85-88.

[24] J. K. Mackenzie, Second paper on statistics associated with the random disorientation of cubes, Biometrika 45 (1/2) (1958) 229-240.

[25] A. Reinhold, H. Briesen, Convex geometry for the morphological modeling and characterization of crystal shapes, Part. Part. Syst. Charact. 28 (3-4) (2011) 37-56.

[26] C. Borchert, E. Temmel, H. Eisenschmidt, H. Lorenz, A. SeidelMorgenstern, K. Sundmacher, Image-based in situ identification of face specific crystal growth rates from crystal populations, Cryst. Growth. Des. 14 (3) (2014) 952-971.

[27] H. Eisenschmidt, A. Voigt, K. Sundmacher, Face-Specific Growth and Dissolution Kinetics of Potassium Dihydrogen Phosphate Crystals from Batch Crystallization Experiments, Cryst. Growth Des. 15 (1) (2015) 219-227.

[28] T. Kovačević, V. Wiedmeyer, J. Schock, F. Pfeiffer, A. Voigt, K. Sundmacher, H. Briesen, Orientation of primary particles in potash alum aggregates, BIWIC 2016, Magdeburg. 
[29] J. W. Mullin, J. Garside, R. Unahabhokha, Diffusivities of ammonium and potassium alums in aqueous solutions, J. appl. Chem. 15 (11) (1965) 502505.

[30] R. C. Gonzalez, R. E. Wood, S. L. Eddins, Digital Image Processing Using Matlab, McGraw Hill Education (India), Gatesmark, LLC, 2010.

[31] Fraunhofer ITWM, Mavi - modular algorithms for volume images, v. 1.4.1.

[32] X. Perez-Sala, L. Igual, S. Escalera, C. Angulo, Uniform Sampling of Rotations for Discrete and Continuous Learning of 2D Shape Models, in: Robotic Vision: Technologies for Machine Learning and Vision Applications, 2013, pp. 23-42.

[33] MathWorks, Matlab, versions 2015b, r2014a.

[34] A. Reinhold, Crystal shape modeling and convex geometry - analysis of geometric state spaces, Ph.D. thesis, Technische Universität München (2015).

[35] K. Fukuda, cddlib, version 094g (2012).

URL https://www . inf .ethz.ch/personal/fukudak/cdd_home/

[36] P. Hammer, Marching cubes (2011).

URL http://www.mathworks.com/matlabcentral/fileexchange/ 32506-marching-cubes/content/MarchingCubes.m

[37] G. Fernàndez, M. Kunt, J.-P. Zrÿd, A new plant cell image segmentation algorithm, in: Image Analysis and Processing. 8th International Conference, ICIAP ‘95. Proceedings, 1995, pp. 229-34.

[38] C. Indhumathi, Y. Y. Cai, Y. Q. Guan, M. Opas, An automatic segmentation algorithm for $3 \mathrm{~d}$ cell cluster splitting using volumetric confocal images, J. Microsc. 243 (1) (2011) 60-76.

[39] C. Zhang, C. Sun, T. D. Pham, Segmentation of clustered nuclei based on concave curve expansion, J. Microsc. 251 (1) (2013) 57-67. 
[40] L. Vincent, P. Soille, Watersheds in digital spaces - an efficient algorithm based on immersion simulations, IEEE Trans. Pattern Anal. Mach. Intell. 13 (6) (1991) 583-598.

[41] P. S. Umesh Adiga, B. B. Chaudhuri, An efficient method based on watershed and rule-based merging for segmentation of 3-D histo-pathological images, Pattern Recognit. 34 (7) (2001) 1449-1458.

[42] F. Long, H. Peng, E. Myers, Automatic segmentation of nuclei in 3D microscopy images of C.elegans, in: 2007 4th IEEE International Symposium on Biomedical Imaging : Macro to Nano, Vols 1-3, Arlington, VA, APR 12-15, 2007, 2007, pp. 536-539.

[43] P. Hough, Methods and means for recognizing complex patterns (US Patent 3069654, 1962).

[44] D. Borrmann, J. Elseberg, K. Lingemann, A. Nüchter, The 3d hough transform for plane detection in point clouds: A review and a new accumulator design, 3D Res. 2 (2) (2011) 32:1-32:13.

[45] J. K. Mackenzie, M. J. Thomson, Some Statistics Associated with the Random Disorientation of Cubes, Biometrika 44 (1-2) (1957) 205-210.

[46] J. K. Mason, C. A. Schuh, The generalized mackenzie distribution: Disorientation angle distributions for arbitrary textures, Acta Mater. 57 (14) (2009) $4186-4197$. 